\title{
A different kind of rising star
}

The Leader in the previous issue alluded that this Leader would focus on the Rising Star palaeontological programme at the new research site in the Cradle of Humankind. Various processes at Nature have delayed the release of the initial findings related to the programme and so, instead, this Leader looks at the emergence of a different rising star: the South African Journal of Science (SAJS).

The SAJS was first published in 1905 and has evolved through the contributions of a number of publishers, editors, editorial staff members and editorial boards. The 110th anniversary of SAJS in 2014 witnessed some stellar indicators of the Journal's performance and scientific calibre as well as its impact, reach and visibility. The Web of Science impact factor for SAJS rose from 0.84 in 2013 to 1.03 in 2014, placing it at 20th in the Web of Science ranking of 55 multidisciplinary journals. That list is topped, and skewed, by Nature and Science, suggesting that 20th place is not, in fact, an unenviable position.

However, a growing number of experts in bibliometrics is coming to the carefully determined conclusion that impact factors are a misleading indicator of quality (as articles often are cited for negative rather than positive reasons), and that journal rejection rates are a far more reliable indicator of quality. In this respect, too, the SAJS performs exceptionally well, with an overall rejection rate of $89 \%$ for all 648 submissions received during 2014.

The Journal also ranked second, at $14 \%$ - marginally behind the South African Medical Journal at 16\% - of total visits to all the titles on the SciELO SA platform. Together with the South African Journal of Education and Water SA, at $13 \%$ and $12 \%$, respectively, these top four journals receive more than half $(55 \%)$ of all visits to the 51 South African journals on the platform.

During the course of 2014, there were 44500 visits to the Journal's website (excluding the submission site), of which 32000 were new visitors - an increase of $18 \%$ over the previous year. About $40 \%$ of visits to the website emanate from South Africa. The average daily number of visits almost doubles on days on which the Table of Contents are distributed electronically. The article receiving the most visits in 2014 - which was published online on only 26 November 2014 - received nearly 1000 visits by the end of 2014 . Forthcoming developments on the website will include a more comprehensive set of impact indicators at article level, which will enable numerous ways to assess online impact.

To enhance its visibility and accessibility, the Journal is indexed by various local and international aggregators. We do not have SAJS usage data for all these sites, but a well-known local platform - the Sabinet Reference platform - gives an indication of the extent of the visibility of SAJS via these databases. During 2014, there were 53000 views of SAJS articles via Sabinet's SA ePublications Collection. The SAJS article downloaded most frequently in 2014 was downloaded 1015 times.

In May 2014, an entirely new development for SAJS moved its presence into the realm of 'social media', giving the Journal a greater online presence than ever before - an important development if the Journal is to capture, hold and develop the interest of young and emerging scholars. In a few short months, the Journal has gained almost 200 Twitter followers and just over 400 Likes on Facebook.

Another new development during the anniversary year was that of a new reading format - an 'easy-to-read' online form of the entire issue was established that allows for page swipes (http://issuu.com/sajs), ideal for browsing on tablets and other mobile devices.

Of course, the reality is that numbers are just numbers. And as William Cameron (and not Albert Einstein as some believe) notably pointed out: 'Not everything that can be counted counts, and not everything that counts can be counted.' And so, going forward, the focus will continue to be on what content is accepted, rather than what the Journal's impact factor is.

That said, for quality content to have an impact, it needs to be as visible and widely accessible as possible. So the endeavour for a visible and well-respected journal that effectively serves the interests of science and scholarship continues. 